\title{
Prevention and management of hyperkalemia in patients treated with renin-angiotensin- aldosterone system inhibitors
}

\author{
Jordan Weinstein MD, Louis-Philippe Girard MD MBT, Serge Lepage MD, Robert S. McKelvie MD PhD, \\ Karthik Tennankore MD SM
}

Cite as: CMAJ 2021 December 6;193:E1836-41. doi: 10.1503/cmaj.210831

$\mathbf{H}$ yperkalemia, defined as a serum potassium level of $5.0 \mathrm{mmol} / \mathrm{L}$ or greater, can lead to severe electrophysiological disturbances, including cardiac arrythmias, that increase morbidity and risk of death. ${ }^{1}$ It is common in patients with conditions that impair potassium excretion by the kidneys, such as chronic kidney disease (CKD), heart failure, hypertension that is difficult to control, diabetes or combinations of these conditions. ${ }^{1,2}$ These patients are commonly treated with renin-angiotensinaldosterone system (RAAS) inhibitors to help lower their risk of CKD progression and cardiovascular events. ${ }^{3}$ However, these medications may trigger or exacerbate hyperkalemia. ${ }^{4-7}$ Some guidelines now recommend initiating and up-titrating these medications to the highest approved dose that the patient can tolerate to optimize clinical outcomes. ${ }^{8,9}$ Therefore, anticipation and prompt management of hyperkalemia is crucial for patients prescribed these medications. We discuss strategies to mitigate the risk of chronic hyperkalemia and to optimize care of patients being treated for CKD, heart failure or associated conditions, as informed by original research, reviews and clinical practice guidelines (Box 1).

\section{Who is at risk of hyperkalemia?}

Conditions that interfere with the kidney's ability to excrete potassium contribute to the development of hyperkalemia because potassium homeostasis is largely achieved by renal excretion. A population-based cohort study found that $28 \%$ of patients with CKD and $39 \%$ of patients with heart failure had at least 1 episode of hyperkalemia. ${ }^{10}$ However, the prevalence of hyperkalemia, as well as the risk of recurrence, increases with a higher burden of comorbid conditions. ${ }^{2,11}$

Other important risk factors include advanced age and the use of medications known to increase serum potassium levels. ${ }^{4}$ Among the medications that lead to hyperkalemia, the most clinically important are RAAS inhibitors, which include angiotensin-converting-enzyme inhibitors, angiotensinreceptor blockers and mineralocorticoid receptor antagonists. ${ }^{4-6} \mathrm{Although}$ there is heterogeneity among studies, a systematic review and meta-analysis of 20 studies found that hyperkalemia is nearly twice as common (risk ratio $1.89,95 \%$ confidence interval 1.56-2.30) in patients on drugs that act on

\section{Key points}

- Chronic hyperkalemia is prevalent among patients with chronic kidney disease, heart failure or other conditions treated with renin-angiotensin-aldosterone system (RAAS) inhibitors, and has been associated with severe adverse outcomes, including death.

- The risk of chronic hyperkalemia may be mitigated by reducing dietary potassium, careful dosing of hyperkalemia-inducing medications, using diuretics that promote renal extraction of hyperkalemia and using potassium binders.

- Accumulating evidence suggests that the adverse effects of reducing or stopping RAAS inhibitors outweigh the potential harms of hyperkalemia, and guidelines recommend maintaining optimal RAAS inhibitor dosing by treating hyperkalemia using other strategies.

- More research is needed to support the prevention and management of hyperkalemia among patients with chronic kidney disease or heart failure.

\section{Box 1: Evidence used in this review}

We conducted a targeted search of MEDLINE to identify original research and review articles on hyperkalemia published through February 2021. Medical subject heading search terms included "hyperkalemia," "chronic kidney disease," "heart failure" and "management." In addition, we reviewed current European, American and Canadian guidelines on the management of hyperkalemia.

the RAAS. ${ }^{5}$ Moreover, data from clinical trials have also shown an increase in the incidence of hyperkalemia in patients with CKD or heart failure on RAAS inhibitor therapy. ${ }^{12}$

An episode of hyperkalemia puts patients at risk of a second episode. An analysis of population-based registries found that second episodes of hyperkalemia were documented within 6 months of the initial occurrence in $40 \%$ of patients with CKD, $49 \%$ of patients with heart failure, and $37 \%$ of patients using RAAS inhibitors. ${ }^{10}$ Predictors of recurrence included a moderate-tosevere first episode of hyperkalemia (potassium $\geq 5.6 \mathrm{mmol} / \mathrm{L}$ ), low estimated glomerular filtration rate (GFR), diabetes and use of spironolactone. ${ }^{10}$ 


\section{What are the consequences of hyperkalemia?}

Although it is well established that severe hyperkalemia is associated with an increased risk of death, even mild increases in potassium that are often deemed nonemergent may be of clinical importance. There is a "u-shaped" association between serum potassium levels and risk of death, with lowest risk of all-cause death in patients with potassium levels between 4.0 and less than $5.0 \mathrm{mmol} / \mathrm{L} .{ }^{11} \mathrm{~A}$ retrospective cohort study from Manitoba found that, compared with patients with normal potassium levels, those with hyperkalemia (potassium $\geq 5.0 \mathrm{mmol} / \mathrm{L}$ ) were at increased risk of all-cause death (hazard ratio [HR] 1.15, 95\% confidence interval [Cl] 1.13-1.18), cardiovascular events (HR 1.20, 95\% Cl 1.14-1.26) and death within 30 days (odds ratio [OR] 1.29, 95\% Cl 1.24-1.34). ${ }^{13}$

In Canada, emergency department use is high among patients with CKD. Observational studies have associated hyperkalemia with increased hospital (OR 1.71, 95\% Cl 1.68-1.74) and intensive care unit admissions (OR 3.48, 95\% $\mathrm{Cl} 3.34-3.62$ ), and have found that hyperkalemia accounts for $48 \%$ of all ambulatory care-sensitive conditions among patients on dialysis. ${ }^{13,14}$ Therefore, unsurprisingly, hyperkalemia is associated with substantial costs from frequent hospitalizations for recurrent episodes. ${ }^{13,15}$

Physicians frequently respond to an episode of hyperkalemia by reducing or stopping a patient's RAAS inhibitor regimen. ${ }^{14,16,17}$ However, submaximal dosing of RAAS inhibitors in patients with CKD or heart failure is associated with increased outpatient office visits and overall health care costs, and worse cardiorenal outcomes. ${ }^{16,18}$ This highlights the challenge of attempting to balance the cardiorenal benefits of RAAS inhibitor treatment with the associated risk of chronic hyperkalemia in patients who will benefit most from these drugs.

\section{How often should clinicians assess patients' potassium levels?}

Patients at risk of chronic hyperkalemia may be asymptomatic, and frequent monitoring will increase detection of the condition. ${ }^{19} \mathrm{How}$ ever, since many factors influence the risk of developing hyperkalemia, clinicians should consider several factors when deciding how frequently to test, particularly when initiating treatment with RAAS inhibitors (Box 2). Moreover, practices regarding the appropriate level of hyperkalemia at which to treat are not well standardized. Thresholds for treatment outlined in current guidance are based on averages of patients in a given laboratory system and the "u-shaped" association between serum potassium levels and risk of death. ${ }^{11}$

A rapid rise in potassium is more likely to lead to cardiac arrhythmias than a gradual increase so, when assessing a new result, it is important to consider how quickly the hyperkalemia has developed. ${ }^{21}$

Hyperkalemia in people with a preserved GFR is uncommon and is generally associated with pseudohyperkalemia, in which in vitro hemolysis leads to elevated potassium levels. Repeat testing to rule out pseudohyperkalemia should be considered unless the benefits of verification are outweighed by the potential harm of delaying treatment. ${ }^{19}$

Patient education, nursing support, follow-up (in person or by telephone), self-monitoring or point-of-care testing may facilitate regular monitoring and help ensure timely and effective treatment. ${ }^{20}$
Box 2: Suggested frequency of potassium monitoring $8,19,20$

Patients being considered for treatment with angiotensinconverting-enzyme inhibitors or angiotensin-receptor blockers:

- At treatment initiation

- 1-2 weeks after initiation

- 1-4 weeks after final dose titration

- At regular intervals thereafter based on the patient's individual risk

Patients being considered for treatment with mineralocorticoid receptor antagonists:

- 1 and 2 weeks after initiating or increasing dose

- At 8 and 12 weeks after initiation or increasing dose

- At 6, 9, and 12 months after initiation or increasing dose

- At regular intervals thereafter based on the patient's individual risk

\section{How to manage patients at risk of hyperkalemia}

Figure 1 outlines an approach to the management of patients with hyperkalemia, which can be undertaken in the outpatient setting if resources allow. Beyond emergency treatment of hyperkalemia, experts suggest preventive approaches, such as encouraging patients to eat a low-potassium diet and to stop using supplements containing potassium. Other approaches include treating hypertension associated with heart failure with loop or thiazide diuretics, carefully dosing RAAS inhibitors and any other medications that may elevate potassium to balance potential benefits and risks, correcting concurrent acidosis, and using potassium binders. ${ }^{19}$

\section{Reducing dietary potassium}

Although guidelines recommend that patients at risk of hyperkalemia, in particular those with advanced CKD, decrease the amount of potassium in their diet, few trials have directly studied the impact of dietary potassium restriction in patients with CKD. ${ }^{9,19,22}$ Nonetheless, given the increased risk of chronic hyperkalemia for patients with CKD and heart failure, clinicians should consider referring these patients to a dietitian for guidance. The potential benefit of consuming a low-potassium, predominantly plant-based diet is an important area for future study.

\section{Judicious use of diuretics}

Diuretics, especially loop diuretics, are commonly used to control volume overload and prevent increases in serum potassium. The efficacy of diuretics to reduce serum potassium depends on a patient's kidney function; guidelines recommend this intervention when diuresis or an additional antihypertensive agent is desired. ${ }^{19}$ Use of diuretics can precipitate acute kidney injury and electrolyte abnormalities, however, and the hypokalemic response to diuretics is diminished with low estimated GFR. ${ }^{3,8}$ Diuretics may not be ideal for the long-term maintenance of serum potassium levels, especially for patients with no other compelling reason for their use (i.e., extracellular volume overload). 


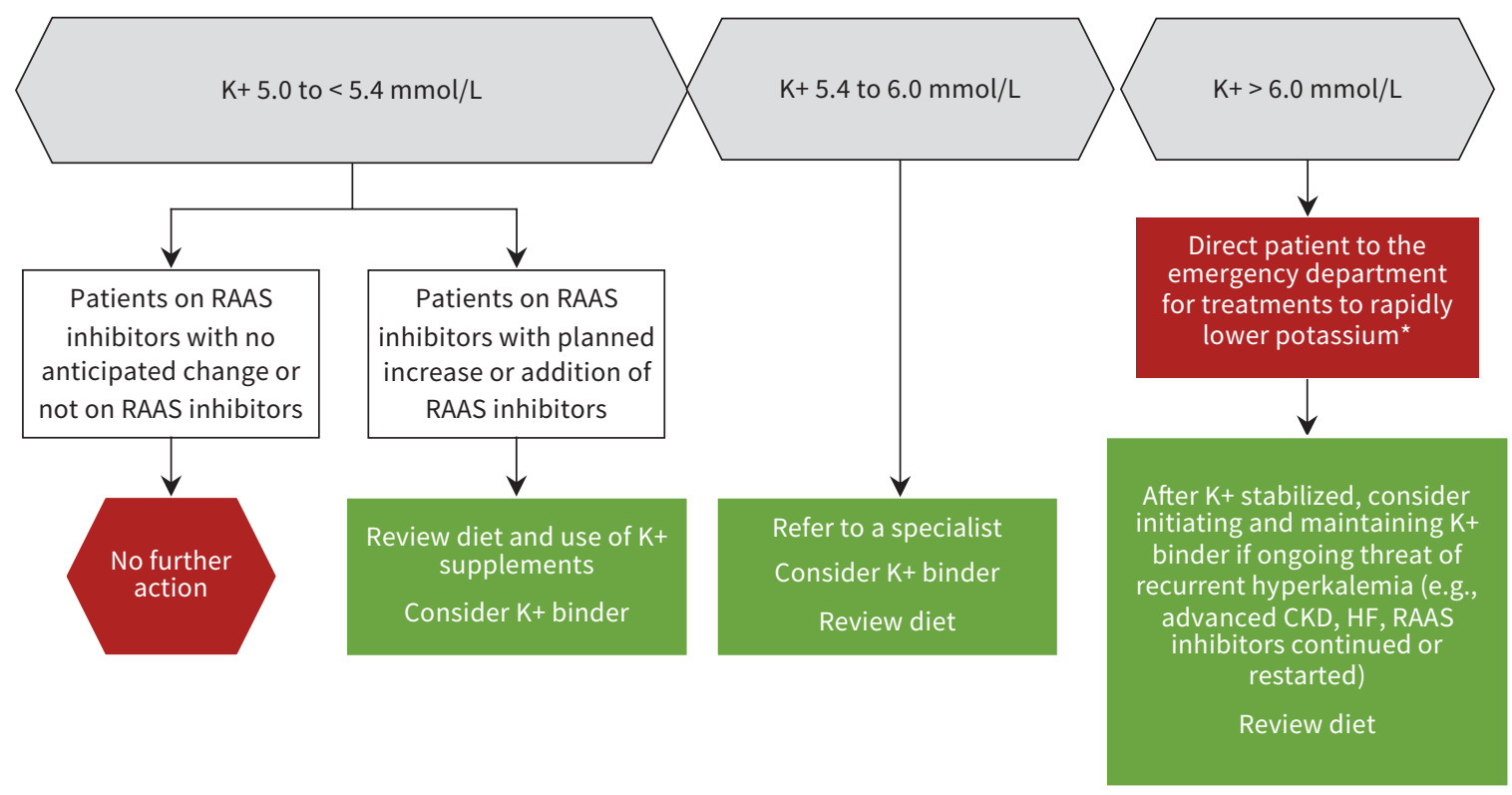

Figure 1: Strategies to mitigate the risk of hyperkalemia and enable more optimal care. Where applicable, clinicians should correct metabolic acidosis with oral sodium bicarbonate (if not contraindicated), promote potassium loss with diuretics in patients with volume overload (if not contraindicated) and consider the use of potassium binders, if available. *Although select patients with potassium values minimally elevated above $6.0 \mathrm{mmol} / \mathrm{L}$ can be managed as outpatients, given the variable availability of resources to rapidly treat and remeasure potassium in a timely fashion, management in the emergency department is recommended. Details of acute management are beyond the scope of this review. Note: CKD $=$ chronic kidney disease, $\mathrm{HF}=$ heart failure, $\mathrm{K}+=$ potassium, $\mathrm{RAAS}=$ renin-angiotensin-aldosterone system.

\section{Use of sodium bicarbonate}

Treatment with oral sodium bicarbonate promotes potassium elimination through increased urinary potassium excretion. It can be used effectively to reduce the risk of hyperkalemia in patients with CKD and metabolic acidosis. However, in patients who develop volume overload, sodium bicarbonate may need to be stopped. ${ }^{8,19}$

\section{Careful dosing of RAAS inhibitors}

Reducing and stopping medications that induce hyperkalemia such as RAAS inhibitors - are commonly used strategies for preventing recurrent episodes of hyperkalemia., ${ }^{3,19,22}$ However, given evidence that the benefits of RAAS inhibitors outweigh their potential harms, including hyperkalemia, recent guidelines now recommend that clinicians maintain an evidence-based dose of RAAS inhibitors. Lowering the dose to reduce the likelihood of recurrent hyperkalemia should be undertaken as a last resort after attempting measures to maintain the evidence-based dose of RAAS inhibitors, including using potassium binders.

\section{Use of potassium binders}

Sodium polystyrene sulfonate, sodium zirconium cyclosilicate and patiromer are potassium binders approved for use in Canada. ${ }^{8,9,23-25}$ For many years, sodium polystyrene sulfonate was the only medication specifically indicated for the treatment of hyperkalemia, but it was approved before the introduction of modern regulatory standards, and evidence from randomized trials to support its use is sparse. ${ }^{3} \mathrm{~A}$ small, single-centre study of 33 outpatients with CKD and mild hyperkalemia (5.0-5.9 mmol/L) found that sodium polystyrene sulfonate was superior to placebo in the reduction of serum potassium levels at 1 week (mean difference between groups $-1.04 \mathrm{mmol} / \mathrm{L}, 95 \% \mathrm{Cl},-1.37$ to $-0.71 \mathrm{mmol} / \mathrm{L}){ }^{26}$ However, the effects of the drug are not predictable and it has been associated with risk of acute bowel necrosis, hypernatremia, diarrhea and gastrointestinal toxicity, particularly with older formulations that contain sorbitol. ${ }^{27}$ One retrospective, matched cohort study found that, over 30 days, use of sodium polystyrene sulfonate was associated with nearly double the incidence of adverse gastrointestinal events than nonuse (37 events [0.2\%] v. 18 events [0.1\%], HR 1.94, 95\% Cl 1.10-3.41)..$^{28}$ Moreover, systemic alkalosis has been reported after administration of sodium polystyrene sulfonate in combination with nonabsorbable cation-donating antacids and laxatives such as magnesium hydroxide and aluminum carbonate; magnesium-containing laxatives should not be used with sodium polystyrene sulfonate. ${ }^{23}$ Thus, clinicians must be cognizant of the potential for serious adverse events when managing hyperkalemia with sodium polystyrene sulfonate. Newer potassium binders should be considered instead, if available.

Sodium zirconium cyclosilicate and patiromer, are newer potassium binders that are generally safer, more predictable and better tolerated by the patient (Table 1). Sodium zirconium cyclosilicate is an orally administered, insoluble, inorganic cation exchanger that selectively captures potassium ions in exchange for hydrogen and sodium ions in the gastrointestinal lumen, thereby increasing potassium fecal excretion and reducing serum potassium. Its efficacy and safety have been shown in phase 2 and 3 clinical trials in both patients receiving dialysis and those not on dialysis. Reported adverse events are generally mild to moderate 
Table 1: Overview of potassium binders

\begin{tabular}{|c|c|c|c|}
\hline Characteristic & $\begin{array}{l}\text { Sodium polystyrene } \\
\text { sulfonate }^{23}\end{array}$ & $\begin{array}{l}\text { Sodium zirconium } \\
\text { cyclosilicate }^{25}\end{array}$ & Patiromer ${ }^{24,29}$ \\
\hline Mechanism & Nonabsorbed cation-exchange resin & $\begin{array}{l}\text { Inorganic cation exchanger with a } \\
\text { crystalline structure that entraps } \mathrm{K}+\end{array}$ & $\begin{array}{l}\text { Nonabsorbed cation-exchanger } \\
\text { polymer that binds } \mathrm{K}+\end{array}$ \\
\hline Site & Colon & Entire GI tract & Colon \\
\hline $\begin{array}{l}\text { Exchange ion for } \\
\text { potassium }\end{array}$ & Sodium & Sodium & Calcium \\
\hline Onset of action & Hours to days & 1 hour & 4 to 7 hours \\
\hline $\begin{array}{l}\text { Enablement of RAAS } \\
\text { inhibitors }\end{array}$ & Not on label or shown & $\begin{array}{l}\text { Not on label } \\
\text { About } 70 \% \text { of patients in phase } 2 \\
\text { and } 3 \text { studies were receiving RAAS } \\
\text { inhibitors at baseline }\end{array}$ & $\begin{array}{l}\text { On label } \\
99.4 \% \text { of patients in phase } 2 \text { and } 3 \\
\text { studies were receiving RAAS } \\
\text { inhibitors at baseline }\end{array}$ \\
\hline Adverse events & $\begin{array}{l}\text { GI stenosis, intestinal ischemia, } \\
\text { ischemic colitis, rectal hemorrhage, } \\
\text { GI necrosis, intestinal perforation } \\
\text { with fatal outcomes, } \\
\text { hypomagnesemia, hypokalemia, } \\
\text { hypocalcemia }\end{array}$ & $\begin{array}{l}\text { Mild-to-moderate GI } \\
\text { effects, edema, hypokalemia } \\
\text { (0\%-11\%, dose-dependent effect) }\end{array}$ & $\begin{array}{l}\text { Mild-to-moderate GI } \\
\text { effects, hypomagnesemia, } \\
\text { hypokalemia }(3 \%-5.6 \%)\end{array}$ \\
\hline Recommended dose & $\begin{array}{l}\text { Oral: } 15 \mathrm{~g}, 1-4 \text { times daily } \\
\text { Rectal: } 30-50 \mathrm{~g}, 1-2 \text { times daily }\end{array}$ & $\begin{array}{l}10 \mathrm{~g} 3 \text { times daily for up to } 48 \text { hours, } \\
\text { then } 5-10 \mathrm{~g} \text { once a day. } \\
\text { No more than } 10 \mathrm{~g} \text { once a day for } \\
\text { maintenance therapy }\end{array}$ & $\begin{array}{l}8.4 \mathrm{~g} \text { once a day. } \\
\text { Dose can be titrated at } \geq 1 \text {-wk } \\
\text { intervals in } 8.4 \mathrm{~g} \text { increments to a } \\
\text { maximum of } 25.2 \mathrm{~g} \text { once a day }\end{array}$ \\
\hline Daily list price and funding & Generic & $\begin{array}{l}\$ 12.50(5 \mathrm{~g}), \$ 25(10 \mathrm{~g}) ; \text { negative } \\
\text { CADTH recommendation* }\end{array}$ & $\begin{array}{l}\$ 13 \text { (flat); positive CADTH } \\
\text { recommendation* }\end{array}$ \\
\hline
\end{tabular}

in severity and are manageable without interruption of treatment. Edema has been reported, possibly a consequence of the drug containing sodium, and patients should be monitored for fluid overload. ${ }^{30-36}$ In clinical trials, $4.1 \%$ of patients treated with sodium zirconium cyclosilicate also developed hypokalemia with a serum potassium value less than $3.5 \mathrm{mmol} / \mathrm{L}$, which was resolved by adjusting the dose or stopping the drug. ${ }^{25}$

Volume overload was not identified as an adverse outcome in trials of patiromer. This drug contains calcium as its gastrointestinal exchange ion and acts in the colon, which means its onset of action is relatively prolonged. The utility of patiromer in treating hyperkalemia has been shown in phase 2 and 3 trials of patients with hyperkalemia, including those with CKD and heart failure on RAAS inhibitors. No serious adverse events have been associated with patiromer, although reported adverse events include non-serious constipation, diarrhea, nausea or vomiting, abdominal discomfort, flatulence and electrolyte disturbances (e.g., hypokalemia and hypomagnesemia). ${ }^{37-40}$

A growing body of evidence supports using the newer potassium binders to facilitate the continuation and optimization of RAAS inhibitors in patients with hypertension, heart failure and CKD. ${ }^{35,37,38,40}$ In a phase 2, double-blind, placebo-controlled trial of patients with heart failure (PEARL-HF), patients randomized to patiromer showed a significantly lower incidence of hyperkalemia $(7.3 \%$ v. $24.5 \%$ on placebo), which enabled greater use of spironolactone at $50 \mathrm{mg} / \mathrm{d}(91 \%$ v. $74 \%) .{ }^{37}$ Similarly, in the AMBER trial of patients with resistant hypertension and CKD, more patients in the treatment arm were able to continue spironolactone with less incidence of hyperkalemia than those on placebo. ${ }^{40}$ Although no study has been specifically designed to assess the RAAS inhibitor-enabling potential of sodium zirconium cyclosilicate, most patients with hyperkalemia enrolled in the ZS-005 study achieved normal potassium levels without substantial changes in their RAAS inhibitor regimen. ${ }^{35}$

Cost and drug plan reimbursement may limit adoption of the new potassium binders. Although the new potassium binders are funded through certain private plans, and the Canadian Agency for Drugs and Technologies in Health has recommended reimbursement of patiromer by public drug plans, it costs about $\$ 3500$ to $\$ 7000$ per patient annually, depending on dose. ${ }^{29}$ Further price negotiations are believed to be underway through the pan-Canadian Pharmaceutical Alliance. Manufacturers and drug plans are urged to find a price that allows access to these drugs for all patients who would benefit from them.

\section{Conclusion}

Hyperkalemia can have serious clinical consequences, including adverse cardiovascular outcomes and death. ${ }^{11}$ Hyperkalemia is associated with RAAS inhibitor therapy, particularly in patients with comorbidities such as CKD and heart failure. ${ }^{1}$ Reduction of RAAS inhibitor dosing may reduce potassium levels, but can have important consequences for optimal disease treatment. ${ }^{16,18}$ 


\section{Box 3: Unanswered questions}

- What are the thresholds of mild, moderate and severe hyperkalemia, and at what threshold should treatment for chronic hyperkalemia be initiated?

- What should be the standard method and frequency for determining potassium levels, and using what reference range?

- Is dietary potassium restriction effective for patients with chronic kidney disease or heart failure who are at risk of hyperkalemia?

- Can newer potassium binders enable continued and optimal treatment with renin-angiotensin-aldosterone system (RAAS) inhibitors to improve important disease outcomes?

Although questions remain on their ability to improve outcomes, treatment with new potassium binders may mitigate the risk of recurrent hyperkalemia by rapidly and sustainably reducing serum potassium levels and enabling more optimal care of patients with guideline-recommended doses of RAAS inhibitors (Box 3 ).

\section{References}

1. Jain N, Kotla S, Little BB, et al. Predictors of hyperkalemia and death in patients with cardiac and renal disease. Am J Cardiol 2012;109:1510-3.

2. Kovesdy CP. Management of hyperkalaemia in chronic kidney disease. Nat Rev Nephrol 2014;10:653-62.

3. Dunn JD, Benton WW, Orozco-Torrentera E, et al. The burden of hyperkalemia in patients with cardiovascular and renal disease. Am J Manag Care 2015;21 (Suppl 15):s307-15.

4. Desai AS, Swedberg K, McMurray JJV, et al. Incidence and predictors of hyperkalemia in patients with heart failure: an analysis of the CHARM Program. J Am Coll Cardiol 2007;50:1959-66

5. Albasri A, Hattle M, Koshiaris C, et al. Association between antihypertensive treatment and adverse events: systematic review and meta-analysis. BMJ 2021; 372:n189. doi: 10.1136/bmj.n189.

6. McMurray JJV, Packer M, Desai AS, et al. Angiotensin-neprilysin inhibition versus enalapril in heart failure. N Engl J Med 2014;371:993-1004.

7. Bakris GL, Agarwal R, Anker SD, et al. Effect of finerenone on chronic kidney disease outcomes in type 2 diabetes. N Engl J Med 2020;383:2219-29.

8. Kidney Disease: Improving Global Outcomes (KDIGO) Diabetes Work Group. KDIGO 2020 clinical practice guideline for diabetes management in chronic kidney disease. Kidney Int 2020;98(4S):S1-115.

9. Kidney Disease: Improving Global Outcomes (KDIGO) Blood Pressure Work Group. KDIGO 2021 clinical practice guideline for the management of blood pressure in chronic kidney disease. Available: https://kdigo.org/guidelines/ blood-pressure-in-ckd/ (accessed 2021 Feb. 25).

10. Adelborg K, Nicolaisen SK, Hasvold P, et al. Predictors for repeated hyperkalemia and potassium trajectories in high-risk patients - a population-based cohort study. PLoS One 2019;14:e0218739.

11. Collins AJ, Pitt B, Reaven N, et al. Association of serum potassium with allcause mortality in patients with and without heart failure, chronic kidney disease, and/or diabetes. Am J Nephrol 2017;46:213-21.

12. Juurlink DN, Mamdani MM, Lee DS, et al. Rates of hyperkalemia after publication of the Randomized Aldactone Evaluation Study. N Engl J Med 2004;351:543-51.

13. Hougen I, Leon SJ, Whitlock R, et al. Hyperkalemia and its association with mortality, cardiovascular events, hospitalizations, and intensive care unit admissions in a population-based retrospective cohort. Kidney Int Rep 2021;6:1309-16.

14. Ronksley PE, Tonelli M, Manns BJ, et al. Emergency department use among patients with CKD: a population-based analysis. Clin J Am Soc Nephrol 2017;12:304-14

15. Kanda E, Kashihara N, Kohsaka S, et al. Clinical and economic burden of hyperkalemia: a nationwide hospital-based cohort study in Japan. Kidney Med 2020;2:742-752.e1.

16. Epstein M, Reaven NL, Funk SE, et al. Evaluation of the treatment gap between clinical guidelines and the utilization of renin-angiotensin-aldosterone system inhibitors. Am J Manag Care 2015;21(Suppl 11):S212-20.
17. Hundemer GL, Talarico R, Tangri N, et al. Ambulatory treatments for RAAS inhibitor-related hyperkalemia and the 1-year risk of recurrence. Clin J Am Soc Nephrol 2021;16:365-73.

18. Polson M, Lord TC, Kangethe A, et al. Clinical and economic impact of hyperkalemia in patients with chronic kidney disease and heart failure. J Manag Care Spec Pharm 2017 Apr;23(4-a Suppl):S2-9.

19. Clase CM, Carrero J-J, Ellison DH, et al. Potassium homeostasis and management of dyskalemia in kidney diseases: conclusions from a Kidney Disease: Improving Global Outcomes (KDIGO) Controversies conference. Kidney Int 2020;97:42-61.

20. Ferreira JP, Butler J, Rossignol P, et al. Abnormalities of potassium in heart failure: JACC State-of-the-Art Review. J Am Coll Cardiol 2020;75:2836-50.

21. Conway R, Creagh D, Byrne DG, et al. Serum potassium levels as an outcome determinant in acute medical admissions. Clin Med (Lond) 2015;15:239-43.

22. Howlett JG, Chan M, Ezekowitz JA, et al. The Canadian Cardiovascular Society heart failure companion: bridging guidelines to your practice. Can J Cardiol 2016;32:296-310.

23. KAYEXALATE [product monograph]. Laval (QC): sanofi-aventis Canada; 2018. Available: https://pdf.hres.ca/dpd_pm/00047478.PDF (accessed 2021 Mar. 31).

24. VELTASSA [product monograph]. Saint-Laurent (QC): Vifor Fresenius Medical Care Renal Pharma; 2019. Available: https://pdf.hres.ca/dpd_pm/00053458. PDF (accessed 2021 Mar. 4).

25. LOKELMA [product monograph]. Mississauga (ON): AstraZeneca Canada; 2020 Available: https://pdf.hres.ca/dpd_pm/00057706.PDF (accessed 2021 Mar. 4).

26. Lepage L, Dufour A-C, Doiron J, et al. Randomized clinical trial of sodium polystyrene sulfonate for the treatment of mild hyperkalemia in CKD. Clin J Am Soc Nephrol 2015;10:2136-42.

27. Pitt B, Bakris GL. New potassium binders for the treatment of hyperkalemia. Hypertension 2015;66:731-8.

28. Noel JA, Bota SE, Petrcich W, et al. Risk of hospitalization for serious adverse gastrointestinal events associated with sodium polystyrene sulfonate use in patients of advanced age. JAMA Intern Med 2019;179:1025-33.

13. Canadian Agency for Drugs and Technologies in Health. CADTH reimbursement recommendation patiromer (Veltassa). Can J Health Technol 2021;1:1-10.

30. Stavros F, Yang A, Leon A, et al. Characterization of structure and function of ZS-9, a K+ selective ion trap. PLoS One 2014;9:e114686.

31. Kosiborod M, Rasmussen HS, Lavin P, et al. Effect of sodium zirconium cyclosilicate on potassium lowering for 28 days among outpatients with hyperkalemia: the HARMONIZE randomized clinical trial. JAMA 2014;312:2223-33.

32. Ash SR, Singh B, Lavin PT, et al. A phase 2 study on the treatment of hyperkalemia in patients with chronic kidney disease suggests that the selective potassium trap, ZS-9, is safe and efficient. Kidney Int 2015;88:404-11.

33. Anker SD, Kosiborod M, Zannad F, et al. Maintenance of serum potassium with sodium zirconium cyclosilicate (ZS-9) in heart failure patients: results from a phase 3 randomized, double-blind, placebo-controlled trial. Eur J Heart Fail 2015;17:1050-6.

34. Packham DK, Rasmussen HS, Lavin PT, et al. Sodium zirconium cyclosilicate in hyperkalemia. N Engl J Med 2015;372:222-31.

35. Spinowitz BS, Fishbane S, Pergola PE, et al. Sodium zirconium cyclosilicate among individuals with hyperkalemia: a 12-month phase 3 study. Clin J Am SoC Nephrol 2019;14:798-809.

36. Fishbane S, Ford M, Fukagawa M, et al. A phase 3b, randomized, double-blind, placebo-controlled study of sodium zirconium cyclosilicate for reducing the incidence of predialysis hyperkalemia. J Am Soc Nephrol 2019;30:1723-33.

37. Pitt B, Anker SD, Bushinsky DA, et al. Evaluation of the efficacy and safety of RLY5016, a polymeric potassium binder, in a double-blind, placebocontrolled study in patients with chronic heart failure (the PEARL-HF) trial. Eur Heart J 2011;32:820-8.

38. Weir MR, Bakris GL, Bushinsky DA, et al. Patiromer in patients with kidney disease and hyperkalemia receiving RAAS inhibitors. N Engl J Med 2015;372: 211-21.

39. Bakris GL, Pitt B, Weir MR, et al. Effect of patiromer on serum potassium level in patients with hyperkalemia and diabetic kidney disease: the AMETHYST-DN randomized clinical trial [published erratum in JAMA 2015;314:731. doi: 10.1001/jama.2015.9237] JAMA 2015;314:151-61.

40. Agarwal R, Rossignol P, Romero A, et al. Patiromer versus placebo to enable spironolactone use in patients with resistant hypertension and chronic kidney disease (AMBER): a phase 2, randomised, double-blind, placebo-controlled trial. Lancet 2019;394:1540-50. 
Competing interests: Jordan Weinstein reports consultancy and advisory work for AstraZeneca, Bayer, BI/Lily, Alexion, Merck, Amgen, Janssen, Otsuka and CPD Network. Louis-Philippe Girard reports consultancy and advisory work for Otsuka and Astra Zeneca. Robert McKelvie reports consultancy and advisory work for Otsuka, AstraZeneca, Bayer, Merck and Novartis. Karthik Tennankore reports consultancy and advisory work for Otsuka, Janssen, Astra Zeneca, Baxter and Bayer. Sodium zirconium cyclosilicate is licensed by AstraZeneca Canada. Patiromer is imported and distributed by Otsuka Canada. No other competing interests were declared.

This article has been peer reviewed.

Affiliations: Division of Nephrology (Weinstein), St. Michael's Hospital, University of Toronto, Toronto, Ont.; Glomerulonephritis Clinic (Girard), University of Calgary, Calgary, Alta.; Sherbrooke University (Lepage), Sherbrooke, Que.; Division of Cardiology (McKelvie), St. Joseph's Health Care; Canada and Western University (McKelvie), London, Ont.; Nova Scotia Health Authority (Tennankore), Dalhousie University, Halifax, NS
Contributors: All of the authors contributed to the conception and design of the work, drafted the manuscript, revised it critically for important intellectual content, gave final approval of the version to be published and agreed to be accountable for all aspects of the work.

Content licence: This is an Open Access article distributed in accordance with the terms of the Creative Commons Attribution (CC BYNC-ND 4.0) licence, which permits use, distribution and reproduction in any medium, provided that the original publication is properly cited, the use is noncommercial (i.e., research or educational use), and no modifications or adaptations are made. See: https:// creativecommons.org/licenses/by-nc-nd/4.0/

Funding: This review was sponsored by an unrestricted education grant from Otsuka Canada.

Acknowledgement: The authors thank Maryssa Canuel PhD MBA of liV Medical Education Agency for editorial support.

Correspondence to: Jordan Weinstein, drjweinstein@gmail.com 